\title{
Surgical Approaches to Cat Breast Cancer (Mammary Tumor), their Treatment and Management at Richmond Crawford Veterinary Hospital Karachi (RCVH), Sindh, Pakistan.
}

\author{
Meena Arif Memon ${ }^{1}$, Farzana Abbasi ${ }^{2}$, Imtiaz Hussain Raja Abbasi ${ }^{3}$, Gulfam Ali \\ Mughal, ${ }^{4}$ Rab Nawaz Soomro, ${ }^{3}$ and $*$ Arif Saleem Memon ${ }^{5}$ \\ 1. Richmond Crawford Veterinary Hospital Karachi (RCVH), Sindh, Pakistan. \\ 2. Shah Abdul Latif University Khairpur, Pakistan. \\ 3. College of Animal Science and Technology, Northwest A \& F University, Yangling, Shaanxi \\ 712100 , China.
}

4. Department of Livestock, Sindh Agriculture University Tandojam.

5. Department of Livestock and Fisheries, Sindh, Pakistan.

*Corresponding Author; nimrani_memon@yahoo.com

\begin{abstract}
A study was done during July to August 2015 at Karachi Division; infected cats were carried at Richmond Crawford Veterinary Hospital Karachi (RCVH), Sindh Pakistan. 10 to 12 years old 08 cats with mammary neoplasm, showed signs \& symptom; swelling on the breast, change in breast form, liquid coming out from the barest and skin becomes dark pink in color, it was confirmed by breast X-ray \& other (blood \& urine) test by help of Aga Khan Hospital Karachi. After confirmation a surgical removal of tumor was done with mastectomy procedure, during operation it was noted that tumor could reach on her vascular wall. Further patients treated with antibiotic and anti-inflammatory medicine recommended to owners. Neomycin Cream, Cicatrin Powder, Hydrogen per oxide, Payodine, Tincture Benzoide, Cotton, mepore; to disinfect the area and then finally make the mixture like a paste apply at wound. Result showed, after seven days wound was healed properly, animal movement and other behaviors were normal noted. The main opinion from research is that to reduce infectious perilous disease in our pets, pets directly infected our children and adult family members.
\end{abstract}

Keywords: cate, mammary neoplasm, mastectomy.

\section{INTRODUCTION}

Breast tumor is the type of over growth of cells that develops from the breast tissue (NCI, 2014). Tumor starts when an abnormal cell starts reproducing and invading the good cells in the body. These abnormal cells often unite in developing a mass known as a tumor. It's important to remember that not all tumors are malignant (cancerous) some can be benign tumors (non-cancerous). Malignant tumors tend to spread through the bloodstream or lymphatic system. There are different symptoms of breast cancer, swelling, puffiness or clumps in the breast, a change in breast form, dimpling of the skin, liquid coming out from the teats, or skin becomes radish in color and scaly (NCI, 2014). In those with distant spread of the disease, there may be swollen lymph nodes, difficult breathing or yellow skin (Saunders et al, 2009) risk factors for developing breast cancer; sex, exercise age and nutrition (NCI, 2014 and World Health Organization, 2014).

Mammary tumors or breast cancers are the third most commonly neoplasia occurs in in cats, following lymphoid and skin cancers (Viste et al, 2002). The incidence of mammary tumors in cats is reduced by $91 \%$ in cats spayed prior to six months of age and by $86 \%$ in cats spayed prior to one year according to (Overley et al, 2005). Siamese cats and Japanese breeds seem to have increased risk (Ito et al, 1996) and obesity also appears to be a factor in tumor development (German, 2006). Malignant tumors make up 80 to $96 \%$ of mammary tumors in cats almost all adenocarcinomas (Millanta et al, 2002). Male cats may also develop mammary adenocarcinoma, albeit rarely and the clinical course is similar to female cats (Skorupski et al, 2005). Cats with tumors less than three $\mathrm{cm}$ had an average survival time of 21 months and cats with tumors greater than three $\mathrm{cm}$ had an average survival of 12 
months. Cancer appears to be age related and is often diagnosed in older dogs or cats because older animals have weaker immune systems thus unable to fight cancer causing cells. Cancer appears to be age related and is often diagnosed in older dogs or cats, older animals have weaker immune systems thus unable to fight cancer causing cells (Viste et al, 2002).

About 10 percent of cat mammary tumors have estrogen receptors, so spaying at the time of surgery has little effect on recurrence or survival time (Morrison et al, 1998). Metastasis tends to be to the lungs and lymph nodes and rarely to bone (Waters et al, 1998). Diagnosis and treatment is similar to the dog. There is a better prognosis with bilateral radical surgery (removing the both mammary chains) than with more conservative surgery (Novosad et al, 2006). Doxorubicin has shown some promise in treatment (Moore et al, 2004).

Considering the importance of pet, the research was carried out to with the following objectives: to solve growing problem of mammary tumor in pets through advance surgical approaches and improve management practice on pets farm for better control of disease, improve performance of vaccination program in Karachi division to prevent outbreak of seasonal and contagious disease; during the study and experiment period.

\section{Objective}

To solve increasing problem of mammary tumor in pets through advance surgical approaches and expand management practice on pet's farms for better control of disease.

\section{Material method}

\section{Case presentation}

Richmond Crawford Veterinary Hospital is located at Jinnah road Karachi near radio Pakistan. Veterinary Hospital mostly provides service of prevention, treatment, surgery, care and management of pets, ornamental animals and birds. They do all practical work in hospital giving best treatment to the patient, aims of Richmond Crawford Veterinary Hospital were to prevent outbreak of pet's disease, to save the life of pet's animals by giving best treatment. They were treating the sick small ruminants and pets by using antibiotics, removing deformities of pets by using advance surgical methods.

\section{Materials}

In order to ascertain the objects under study and arrive at fruitful results; materials and equipment available at Richmond Crawford Veterinary Hospital Karachi (RCVH), were assessed

Antibiotics: Fluconazole, Trobersin Gentamycin, Fluconazole , Almox LA, Oxy LA, Penicillin, Pararock, Metronidazole, Novamox Triberissen, Amoxillin, Clindamycin , Augmentin, Neomycin Cream and symodex (multivitamin).

Antiseptics: Pyodine, Dettol Tergagen spray and Potassium Paramagnet Lotion.

Vaccines: Collibacillosis (E. coli) vaccine, HSV (Hemorrhagic Septicemia Vaccines), ETV (Enterotoxaemia Vaccine), FMD (Foot and Mouth Disease), PPR (pet peptide ruminates) and Rabisin Vaccine.

Powders: Sodium bicarbonate cicatrix (Koalin) boric acid, Cicatrin Powder, Hydrogen Per Oxide, Payodine, Tincture Benzoide, Cotton, mepore etc.

Drenches: Nilverm,, Nilzan plus Ozox plus ,Cyclomate and Zanil.

Biologics Like; sterile diluents, normal saline, distilled water

\section{Instruments for surgical approaches}

Instruments: x-ray machine, blood \& urine test machine, advance microscope, slide, petti dishes, Syringes, Needles, scissors forceps, different suture material, surgical room and small blood test laboratory.

\section{Method of experiment}

Study was done during July to August 2015 at Karachi Division, 30 cats were examined from different area of Karachi city, from which 22 cases was showed different sign and symptoms, having different disease, 08 cases were hypothesized as breast tumor, for confirmation of breast tumor first 
Surgical Approaches to Cat Breast Cancer (Mammary Tumor), their Treatment and Management at Richmond Crawford Veterinary Hospital Karachi (RCVH), Sindh, Pakistan

some investigations were done by help of Aga Khan Hospital laboratory, blood reports and X ray showed breast tumor. History and problem showed signs \& symptom; swelling on the breast, change in breast form, liquid coming out from the barest and skin becomes dark pink in color. Surgical team of (RCV) Hospital was prepared all surgical required material under supervision of Dr. Meena Memon, a surgical removal of cancer/tumor was done with mastectomy procedure, during operation it was noted that cancer could reach on her vascular wall, surgical team were suggested to owner later post operation period for management \& care of wound by clean the surgical area usage of Hydrogen per Oxide, Tincture Benzoide to stop the extra bleeding, payodine to disinfect the area and then finally make the mixture of 50\% Neomycin Cream and 50\% Cicatrin Powder and apply this mixture like a paste evenly on the wound and surrounding the wound three times a day up to healing period, it was noted and tried during operation and post operation period the body temperature, heartbeat and blood pressure remain under control, after operation some antibiotic treatment was under used to normalize temperature and infection, following pictures showed procedure of Mastectomy.

\section{Picture of Mastectomy}

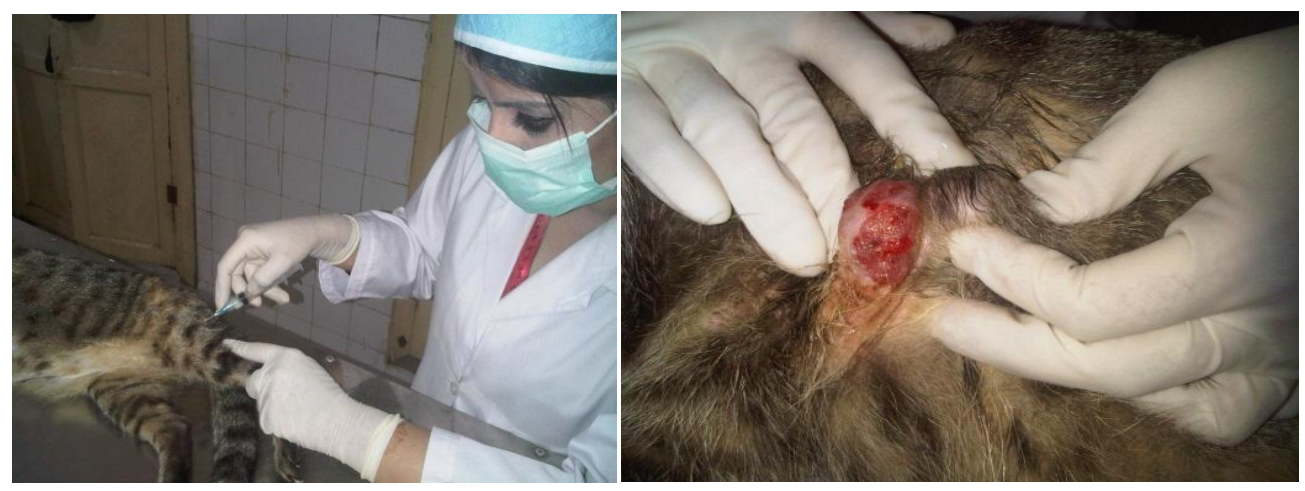

Figur1. Showed anaesthetizing of cat and tumor skin

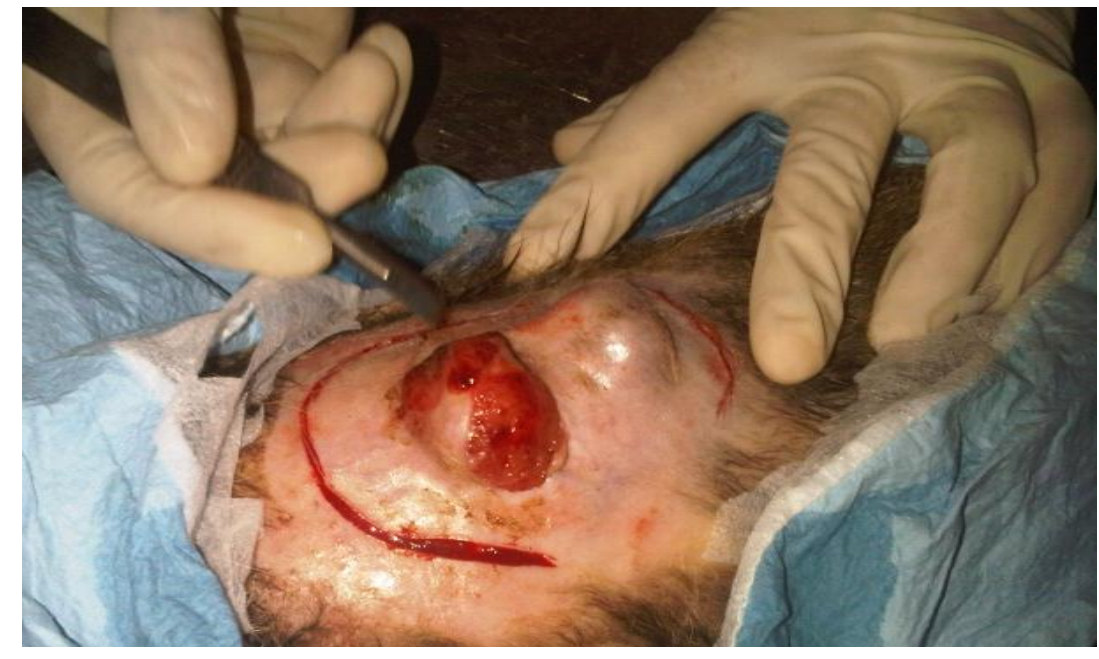

Figure 2: showed skin incision and separation of tumor skin
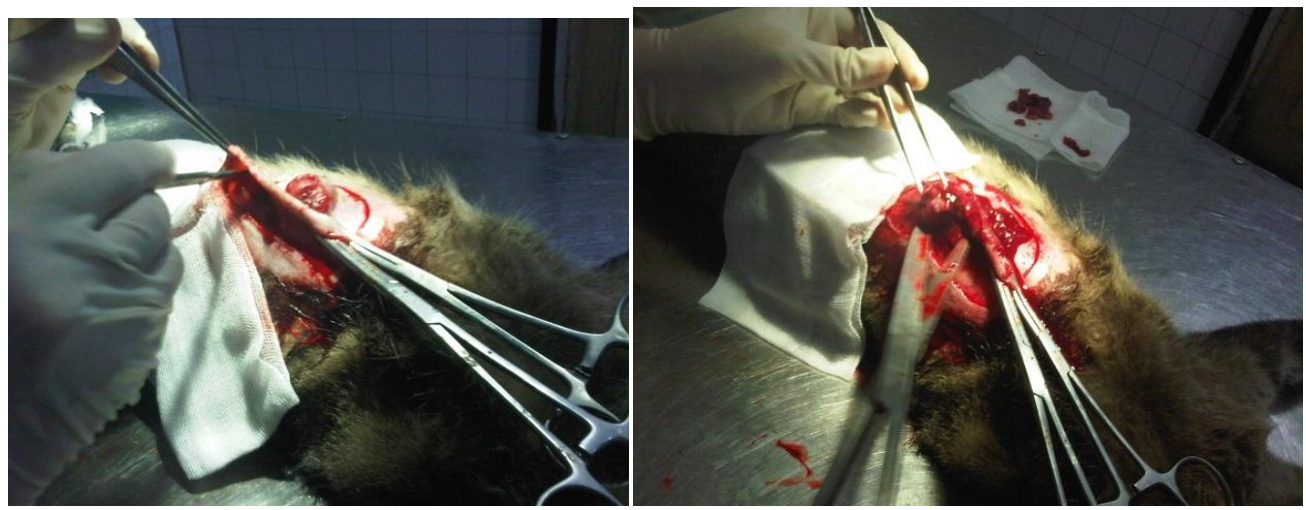

Figure 3. showed surgical removal of tumor cells 


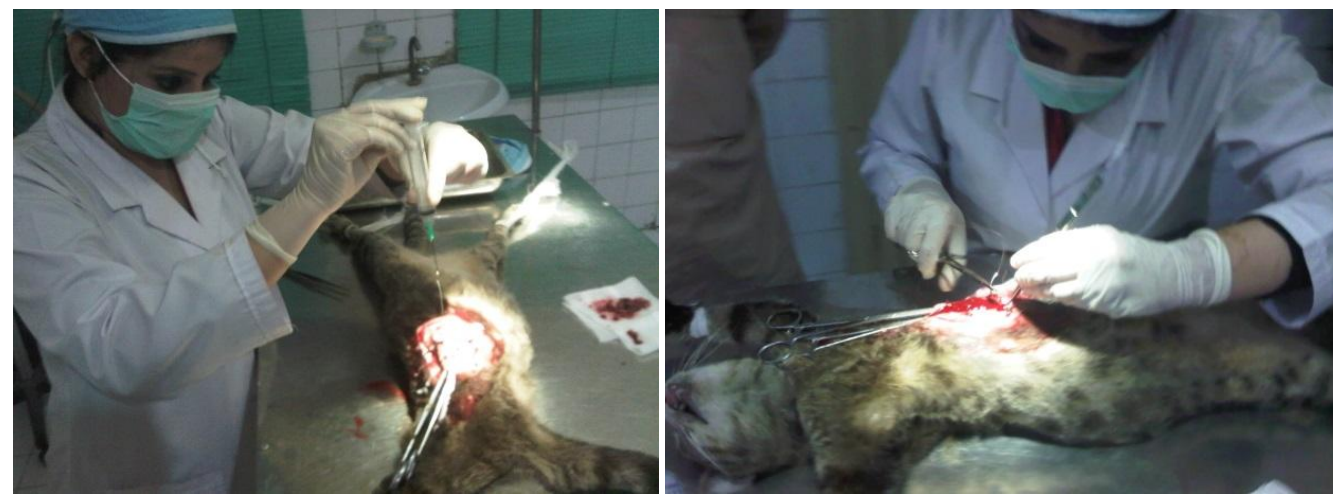

Figure 4. showed spraying antiseptic and suturing
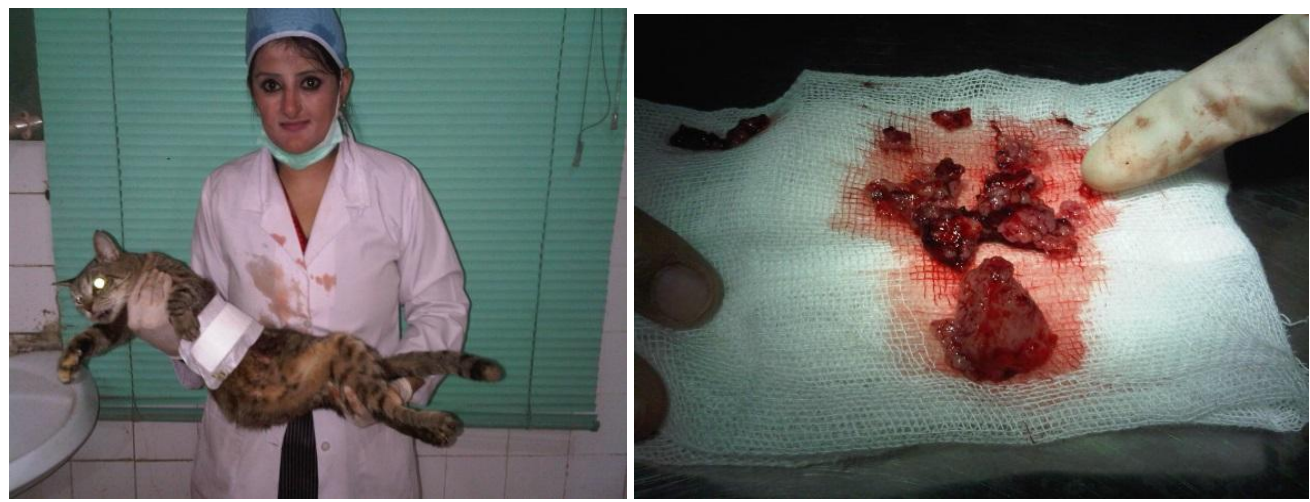

Figure 5. showed cat tumor cells and cat after successful surgery

\section{Postoperative care, suggested and medicine described to patient owners}

Syrup: - Brufen, two times a day (BD) to manage the pain after surgery.

Syrup: - Furplex, once a day (OD), to maintain the loss of blood during surgery.

Syrup: - vi-day line, once a day (OD), to maintain the body condition and supportive medicine with multi vitamins._Treatment was given for Seven (7) days, after seven days was noted that wound was healed properly animal movement and other behaviors were normed.

\section{Discussion}

The United States pet population has been a vastly underutilized resource for cancer therapy studies. Naturally occurring tumors in pet animals develop twice as frequently as in man, have histopathologic features and a biologic behavior similar to tumors in man and progress at a more rapid rate than in man. Cancer of the breast is the most common major cancer in women and the third most commonly reported cancer in cats. Our result and study supported by; MacEwen E. Gregory, 1999 describe that Dogs and Cats develop spontaneous tumors with histopathologic and biologic behavior similar to tumors that occur in humans. The tumors with potential relevance for human cancer biology include osteosarcoma, mammary carcinoma, oral melanoma, oral squamous cell carcinoma, nasal tumors, lung carcinoma, soft tissue sarcomas, and malignant non-Hodgkin's lymphoma. Canine osteosarcoma is a malignant aggressive bone tumor with a $90 \%$ metastasis rate after surgical amputation. Its predictable metastatic rate and pattern and its relative resistance to chemotherapy make this tumor particularly attractive for studying anti-metastasis approaches. Canine and feline malignant mammary tumors are fairly common in middle-aged animals and have a metastatic pattern similar to those in women. Paul W. Ewald, 2011 reported that non-malignant infectious neoplasms occur pervasively in multi cellular life, but oncogenic progression to malignancy is often uncertain, evidence from humans and domestic animals shows that non-malignant infectious neoplasms can develop into cancer. Furthermore: Georges Vlastos, 2004 reported that metastatic breast cancer is generally believed to be associated with a poor prognosis. Therapeutic advances over the past two decades, however, have resulted in improved outcomes for selected patients with limited metastatic disease. Clinical and pathologic characteristics evaluated were age, race, tumor size, stage, pathologic tumor margins, and axillary nodal involvement. Abnormal mammary gland growths are a side effect of progestin therapy 
in some cats. In this retrospective study, the nature and significance of morphologic changes in the mammary gland of 17 megestrol acetate (MA)-treated cats were compared to mammary lesions in 97 untreated cats. Fourteen out of 17 MA-treated cats had non-neoplastic mammary lesions including fibro epithelial hyperplasia (nine cats), lobular hyperplasia (three cat), and duct ectasia (two cats); whereas three MA-treated cats had mammary neoplasms including one adenoma and two carcinomas. Although MA has been causally linked to mammary cancer in cats, only mammary fibro epithelial hyperplasia was clearly associated with MA therapy in this study. Fibro epithelial hyperplasia occurred in older (average age 8.1 years) neutered male and female cats in the MA-treated group and in younger (average age 2.1 years) female cats in the untreated group. Morphologically, both intra ductal and solid fibro epithelial growth patterns were seen. Intra ductal fibro epithelial hyperplasia was further subdivided into papillary and circumferential types. An apparent greater association between MA therapy and the intra ductal types of fibro epithelial hyperplasia was noted.

\section{Conclusion}

The main feedback from our research is that to reduce infectious perilous disease in our pets, pets directly infected our children and adult family members. When take right actions even aggressive, stiff infectious disease can be defeated easily in pets. At RCVH, Karachi Sindh Pakistan our team performed so many surgeries in different animals; it was first case performed in our country Pakistan and successfully treated Mastectomy (Breast Cancer Surgery) in Cat.

\section{ACKNOWLEDGE}

I express my thanks to my Department (Livestock and Fisheries Department, Sindh, Pakistan) and also all staff of Richmond Crawford Veterinary Hospital Karachi (RCVH), who helps me during these complicated cases.

\section{REFERENCES}

Georges Vlastos, MD, David L. Smith, MD, S. Eva Singletary, MD, Nadeem Q. Mirza, MD, MPH, Todd M. Tuttle, MD, Reena J. Popat, BS, Steven A. Curley, MD, Lee M. Ellis, MD, Mark S. Roh, MD, and Jean-Nicolas Vauthey, MD (2004). Long-term Survival after an aggressive surgical approach in patients with breast cancer hepatic metastases. Annals of Surgical Oncology, 11(9): 869-874.

German A (2006). The growing problem of obesity in dogs and cats". J Nutr 136 (7 Suppl): 1940S1946S.

Fidel (1999). Adjuvant therapy for melanoma in dogs: results of randomized clinical trials using surgery, liposome-encapsulated muramyl tripeptide, and granulocyte macrophage colonystimulating factor1. Clinical cancer research. aacrjournals.org; Vol. 5, 4249-4258.

Ito., T, Kadosawa T, Mochizuki M, Matsunaga S, Nishimura R, Sasaki N (1996). Prognosis of malignant mammary tumor in 53 cats". J Vet Med Sci 58 (8): 723-6.

Millanta F, Lazzeri G, Mazzei M, Vannozzi I, Poli A (2002). MIB-1 labeling index in feline dysplastic and neoplastic mammary lesions and its relationship with postsurgical prognosis. Vet Pathol 39 (1): 120-126.

MacEwen, E. G., Carlos O. Rodriguez, Jr., Brenda Phillips, Courtney H. Zwahlen,4 Joyce Obradovich, Robert C. Rosenthal, Leslie E. Fox, Mona Rosenberg, Carolyn Henry, and Janean

Morrison, Wallace B. (1998). Cancer in Dogs and Cats (1st ed.). Williams and Wilkins. ISBN 0-68306105-4.

Moore, Anthony (2004). Advances in the treatment of mammary neoplasia. Retrieved 2007-03-21.

National Cancer Institute (NCI) "Breast Cancer". Retrieved 29 June 2014.

Novosad C, Bergman P, O'brien M, McKnight J, Charney S, Selting K, Graham J, Correa S, Rosenberg M, Gieger T (2006). Retrospective Evaluation of Adjunctive Doxorubicin for the Treatment of Feline Mammary Gland Adenocarcinoma: 67 Cases. J Am Anim Hosp Assoc 42 (2): $110-120$.

Overley B, Shofer F, Goldschmidt M, Sherer D, Sorenmo K (2005). Association between ovary hysterectomy and feline mammary carcinoma. J Vet Intern Med 19 (4):560-3. 
Paul W. Ewald (2011) Evolution of virulence, environmental change, and the threat posed by emerging and chronic diseases. Environmental change, pathogens and human linkages. Ecol Res (2011) 26: 1017-1026.

Saunders, Christobel; Jassal, Sunil (2009). Breast cancer (1. Ed.). Oxford: Oxford University Press. p. Chapter 13. ISBN 978-0-19-955869-8.

Skorupski K, Overley B, Shofer F, Goldschmidt M, Miller C, Sørenmo K (2005). Clinical characteristics of mammary carcinoma in male cats. J Vet Intern Med 19 (1): $52-5$.

Viste J, Myers S, Singh B, Simko E (2002). Feline mammary adenocarcinoma: tumor size as a prognostic indicator. Can Vet J 43 (1): 33-7.

Waters D, Honeckman A, Cooley D, DeNicola D (1998). Skeletal metastasis in feline mammary carcinoma: case report and literature review. J Am Anim Hosp Assoc 34 (2): 103-8.

World Cancer Report 2014. World Health Organization. 2014. pp. Chapter 5.2. ISBN 92-832-0429-8. 